\title{
Sacral neuromodulation outcomes for the treatment of refractory idiopathic detrusor overactivity stratified by indication: Lack of anticholinergic efficacy versus intolerability
}

\author{
Tanya Davis, MD; Iryna Makovey; Michael L. Guralnick MD, FRCSC; R. Corey O'Connor, MD \\ Department of Urology, Medical College of Wisconsin, Milwaukee, Wisconsin
}

See related commentary by Magdy Hassouna at cuaj.ca

Cite as: Can Urol Assoc J 2013;7(5-6):176-8. http://dx.doi.org/10.5489/cuaj.11251. Epub 2012 Sept 10.

\begin{abstract}
Introduction: Patients may fail oral overactive bladder therapies due to either poor drug efficacy or intolerability. We determined if the success of sacral neuromodulation varies if performed secondary to lack of anticholinergic efficacy versus drug intolerability.

Methods: A retrospective review was performed on 152 patients undergoing staged sacral neuromodulation from 2004 to 2010 for refractory idiopathic detrusor overactivity with or without urge incontinence. Outcomes following sacral neuromodulation trials were compared based on the primary indication for anticholinergic failure: lack of drug efficacy versus intolerable side effects.

Results: Overall, successful sacral neuromodulation trials were reported in $70 \%(106 / 152)$ of patients. Successful outcomes were noted in $70 \%(89 / 128)$ and $71 \%(17 / 24)$ of patients with poor anticholinergic efficacy and drug intolerability, respectively ( $p=N S$ ). Conclusions: We found no significant difference in outcome success in patients undergoing sacral neuromodulation trials for refractory detrusor overactivity due to lack of anticholinergic efficacy versus intolerability.
\end{abstract}

\section{Introduction}

Initial management of overactive bladder (OAB) often involves behavioural modification and anticholinergic medications. Anticholinergic medications competitively inhibit muscarinic receptors in the detrusor and urothelium to suppress overactive detrusor contractions and decrease sensation. ${ }^{1}$

Pharmacotherapy, however, is not successful for all patients due to either poor drug efficacy or intolerable side effects, such as dry mouth and constipation, secondary to muscarinic blockade elsewhere in the body., ${ }^{2,3}$ When initial conservative therapy fails, surgical therapies such as sacral neuromodulation (SNM) or intradetrusor injections of botulinum toxin A (BTX-A) may be employed. One of the main mechanisms of action of intradetrusor BTX-A is the prevention of presynaptic acetylcholine neurotransmitter release at the neuromuscular junction. Thus, like anticholinergic medications, intradetrusor BTX-A involves the blockade of acetylcholine neurotransmission in the bladder. We previously found that more patients responded to intradetrusor BTX-A if their indication for the procedure was anticholinergic intolerability as opposed to lack of anticholinergic efficacy, and hypothesized that the similar mechanisms of action may have partly explained this finding. ${ }^{4}$ SNM, a proven secondline treatment for $\mathrm{OAB}$ symptoms in patients refractory to anticholinergic drugs, ${ }^{5}$ is postulated to act by suppressing or enhancing the sacral afferent and efferent bladder nerves to modulate storage and voiding reflexes. ${ }^{6}$ It has a different mechanism of action compared to anticholinergic medications and intradetrusor BTX-A. The purpose of this study is to evaluate our outcomes with SNM for refractory OAB stratified by indication: lack of anticholinergic efficacy versus drug intolerability. Because of the differing mechanisms of action, we hypothesize that the difference in response rates that we previously noted with intradetrusor BTX-A will not be noted with SNM.

\section{Methods}

After institutional review board approval, a retrospective chart review was performed for all patients undergoing staged SNM (InterStim, Medtronic Inc., Minneapolis, MN) at a single tertiary care centre from 2003 to 2010 for the management of idiopathic $\mathrm{OAB}$ symptoms refractory to behavioural modification and anticholinergic medications. All patients were evaluated with complete history and physical examination, urinalysis, post-void bladder ultrasound and video urodynamic studies. Inclusion criteria included video urodynamic studies proven detrusor overactivity, negative urinalysis and failure of at least two or more separate fourweek trials of oral anticholinergic medications at maximally approved dosages. Exclusion criteria were age $<18$ years, 
incomplete bladder emptying measuring $>100 \mathrm{~mL}$, bladder outlet obstruction, impaired bladder compliance, primary neurologic disease, previous pelvic radiotherapy or history of bladder outlet surgery.

Staged SNM was performed in the previously reported fashion, ${ }^{7}$ with unilateral or bilateral tined S3 lead placement. A two-week trial period of unilateral lead stimulation was used to assess efficacy. Trial stimulation was deemed successful if a $>50 \%$ improvement in OAB symptoms was noted based on objective and subjective measures, including 24-hour bladder diary, 24-hour pad weights and patientreported symptom improvement.

Patients were divided into two cohorts based on the patient-reported primary reason for anticholinergic failure: lack of medication efficacy or intolerability due to drug side effects. The outcomes were statistically analyzed using either Student's t or Fisher's exact test.

\section{Results}

A total of 152 patients were included in the study. We tallied demographic data, American Society of Anesthesiologists (ASA) classification results and anticholinergic medication usage for the two groups (Table 1). Overall, successful SNM implantation trials were reported in 70\% (106/152) of patients. When divided into cohorts based on reason for medication failure, success rates were $70 \%(89 / 128)$ and $71 \%(17 / 24)$ for patients with poor anticholinergic efficacy and drug intolerability, respectively $(p=N S)$.

Complications were reported by 12/152 (8\%) patients, including prolonged pain at the SNM battery/incision site $(n=6)$ and wound infection $(n=6)$. Prolonged pain over the SNM battery following stage II SNM was initially managed with oral and topical pain medications. Patients that failed conservative treatment underwent surgical repositioning of the SNM battery. SNM infection (four patients following stage I SNM and two patients after stage II SNM) required complete device explantation with delayed reimplantation for all patients.

\section{Discussion}

First-line anticholinergic therapy for the management of overactive bladder may be ineffective for some patients due to poor efficacy or intolerable side effects. Other treatments, including BTX-A injection and SNS, have been shown to be effective, second-line agents for patients with symptoms refractory to medications. ${ }^{8}$ Currently, no published study has explored whether or not the reason for anticholinergic failure can be used to help predict the rate of SNM success. We reviewed the results of stage I SNM implantation for the management of idiopathic detrusor overactivity refractory to anticholinergic medications due to poor drug efficacy versus lack of tolerability. Our findings showed no significant difference in outcomes when comparing patients undergoing SNS trials due to lack of anticholinergic efficacy versus drug intolerability $(70 \%$ vs. $71 \%, p=N S)$.

We previously demonstrated that intradetrusor BTX-A injections are more successful for the treatment of refractory $\mathrm{OAB}$ if performed in patients with anticholinergic intolerability rather than poor drug efficacy. ${ }^{4}$ BTX-A and anticholinergic drugs both decrease detrusor contractions by preventing acetylcholine from reaching the muscarinic receptors. As a result of similar mechanisms of action, we suspected that patients who responded to anticholinergic medications may be more likely to respond to BTX-A. The current study did not support the same findings. We postulated that the results may be due to the differing mechanisms of action of anticholinergic medications and SNM. Anticholinergic drugs primarily prevent detrusor contraction and decrease bladder sensation by blocking muscarinic receptors in the detrusor and urothelium. ${ }^{1}$ SNM is believed to target the afferent and efferent bladder nerves, modulate the balance between bladder inhibition and excitation and, therefore, reduce $O A B$ symptoms. ${ }^{6,9}$ As a result of the differing mechanisms, the success of SNM should not be predicted based on anticholinergic failure.

Success rates of SNM for the management of refractory OAB symptoms range from $53 \%$ to $80 \% .^{10-12}$ Our success rates in this study fall within the published norms. Several authors have demonstrated that advanced patient age, male gender, increased duration of symptoms and the presence of concomitant medical comorbidities decrease the likelihood of success following SNM. ${ }^{13-16}$ The two cohorts examined in our study were similar in age, gender distribution and overall health. As a result, we do not believe that sampling error contributed to our results. The small sample size and retrospective design, however, do limit the strength of the findings. Additionally, our described success rates reflect findings from stage I SNM trials, not permanently implanted

Table 1. Patient demographics and anticholinergic medication use

\begin{tabular}{|c|c|c|c|c|}
\hline & Total $(n=152)$ & Lack of efficacy ( $n=128$ ) & Intolerable side effects $(n=24)$ & $p$ value \\
\hline Age in years (range) & $55.1(18-86)$ & $54.9(18-86)$ & $55.8(24-83)$ & NS \\
\hline Gender (F:M) & $125: 27$ & 105:23 & $20: 4$ & NS \\
\hline Average medications attempted (range) & $3.0(2-6)$ & $3.0(2-6)$ & $3.1(2-6)$ & NS \\
\hline ASA classification (range) & $2.2(1-4)$ & $2.2(1-4)$ & $2.1(1-4)$ & NS \\
\hline
\end{tabular}

F: female; M: male; ASA: American Society of Anesthesiologists; NS: not significant. 
Davis et al.

devices. Our conclusions cannot be extended to long-term results. As such, this is more of a hypothesis-generating study than a conclusive one. Additional prospective studies are warranted.

\section{Conclusions}

No significant difference in outcomes was observed in patients undergoing SNM trials for the management of $\mathrm{OAB}$ due to lack of anticholinergic efficacy versus drug intolerability. The findings may be secondary to different mechanisms of actions between anticholinergics and SNM. Prospective, multicentre trials are needed to strengthen the results.

Competing interests: None declared.

This paper has been peer-reviewed.

\section{References}

1. Andersson KE, Yoshida M. Antimuscarinics and the overactive detrusor-which is the main mechanism of action? Eur Urol 2003;43:1-5. http://dx.doi.org/10.1016/S0302-2838(02)00540-7

2. Benner JS, Nichol MB, Rovner $E S$, et al. Patient-reported reasons for discontinuing overactive bladder medication. BJU Int 2010;105:1276-82. http://dx.doi.org/10.1111/j.1464-410X.2009.09036.x

3. Lawrence M, Guay DR, Benson SR, et al. Immediate-release oxybutynin versus tolterodine in detrusor overactivity - a population analysis. Pharmacotherapy 2000;20:470-5. http://dx.doi.org/10.1592/ phco.20.5.470.35064

4. Makovey I, Davis T, Guralnick ML, et al. Botulinum toxin outcomes for idiopathic overactive bladder stratified by indication - lack of anticholinergic efficacy versus intolerability. Neurourol Urodyn 2011 (in press). http://dx.doi.org/10.1002/nau.21150
5. Herbison GP, Arnold EP. Sacral neuromodulation with implanted devices for urinary storage and voiding dysfunction in adults. Cochrane Database Syst Rev 2009;CD004202.

6. Leng WW, Chancellor MB. How sacral nerve stimulation neuromodulation works. Urol Clin North Am 2005;32:11-8. http://dx.doi.org/10.1016/i.ucl.2004.09.004

7. Spinelli $M$, Giardiello $G$, Gerber $M$, et al. New sacral neuromodulation lead for percutaneous implantation using local anesthesia - description and first experience. J Urol 2003;170:1905-7. http://dx.doi. org/10.1097/01.ju.0000092634.64580.9a

8. Bosch JLHR, Kelleher C, van Kerrebroeck PEV, et al. What treatment should we use if drugs fail for OAB and, what really works after drugs. Neurourol Urodyn 2010;29:658-61. http://dx.doi.org/10.1002/ nau. 20906

9. van der Pal F, Heesakkers JP, Bemelmans BL. Current opinion on the working mechanisms of neuromodulation in the treatment of lower urinary tract dysfunction. Curr Opin Urol 2006;16:261-7. http://dx.doi. org/10.1097/01.mou.0000232047.87803.1e

10. Scheepens WA, van Koeveringe GA, de Bie RA, et al. Urodynamic results of sacral neuromodulation correlate with subjective improvement in patients with overactive bladder. Eur Urol 2003;43:282-7. http:// dx.doi.org/10.1016/S0302-2838(03)00010-1

11. Van Voskuilen AC, Oerlemans DJ, Weil EH, et al. Medium-term experience of sacral neuromodulation by tined lead implantation. BJU Int 2007;99:107-10. http://dx.doi.org/10.1111/i.1464-410X.2006.06508.x

12. Pham K, Guralnick ML, $O^{\prime}$ Connor RC. Unilateral versus bilateral stage I neuromodulator lead placement for the treatment of refractory voiding dysfunction. Neurourol Urodyn 2008;27:779-81. http://dx.doi. org/10.1002/nau.20577

13. Vaarala MH, Tammela TL, Pertrila I, et al. Sacral neuromodulation in urological indications - the Finnish experience. Scand J Urol Nephrol 2011;45:46-51. http://dx.doi.org/10.3109/00365599.2010. 523013

14. Hassouna MM, Ekelini MS. Early versus late treatment of voiding dysfunction with pelvic neuromodulation. Can Urol Assoc J 2007; 1:106-10.

15. Amundsen AA, Romero MG, Jamison, et al. Sacral neuromodulation for intractable urge incontinence - are there factors associated with cure. Urology 2005;66:746-50. http://dx.doi.org/10.1016/i. urology.2005.04.031

16. White WM, Mobley JD III, Doggweiler R, et al. Sacral nerve stimulation for refractory overactive bladder in the elderly population. J Urol 2009; 182:1449-52. http://dx.doi.org/10.1016/i.juro.2009.06.049

Correspondence: Dr. Michael L. Guralnick, Associate Professor of Urology, Medical College of Wisconsin, 9200 W. Wisconsin Ave, Milwaukee, WI 53226; fax: 414-805-0771; mguralni@mcw.edu 